\title{
Higgs Searches at the Tevatron
}

\author{
Eric B. James*广 \\ Fermi National Accelerator Laboratory \\ E-mail: jameseb@fnal.gov
}

We report on the status of Higgs boson searches at the Tevatron collider as of summer 2011. CDF and $D \emptyset$ perform Higgs searches in numerous final states corresponding to different potential production and decay modes of the Higgs in the context of both standard model (SM) production and other theoretical models. Based on current datasets we observe no excesses consistent with a Higgs signal and proceed to set upper limits on the potential production rate. For SM production we are able to exclude the $100-109 \mathrm{GeV} / c^{2}$ and $156-177 \mathrm{GeV} / c^{2}$ mass ranges at $95 \%$ C.L. Prospects for Tevatron search sensitivities using the full $10 \mathrm{fb}^{-1}$ datsets are also discussed.

The 2011 Europhysics Conference on High Energy Physics, EPS-HEP 2011,

July 21-27, 2011

Grenoble, Rhône-Alpes, France

*Speaker.

${ }^{\dagger}$ on behalf of the CDF and D $\varnothing$ Collaborations 


\section{Introduction}

The Higgs field is introduced into the standard model (SM) of particle physics to explain electroweak symmetry breaking and the origin of particle masses. Experimental observation of the Higgs boson would provide direct evidence for this proposed mechanism, and it remains a primary objective of modern particle physics. Current precision electroweak measurements constrain the mass of a SM Higgs boson to be less than $158 \mathrm{GeV} / \mathrm{c}^{2}$ (one-sided 95\% C.L. upper limit) [1] or $185 \mathrm{GeV} / c^{2}$ when incorporating the LEP-2 direct search lower limit of $114.4 \mathrm{GeV} / c^{2}$ [2]. Nextto-next-to leading order (NNLO) SM cross section calculations for Higgs boson production in Tevatron $p \bar{p}$ collisions at $\sqrt{s}=1.96 \mathrm{TeV}$ indicate a potential for excluding or seeing evidence of Higgs boson production in the final $10 \mathrm{fb}^{-1}$ datasets collected by the CDF and D0 experiments. The two multipurpose Tevatron detectors are able to reconstruct all final state particles and topologies resulting from SM Higgs boson production and decay. Data is collected with efficiencies on the order of $85-90 \%$, and integrated luminosities of up to $8.6 \mathrm{fb}^{-1}$ have been analyzed for the Higgs boson searches covered in these proceedings. The ATLAS and CMS experiments also present within these proceedings [3] preliminary Higgs search results based on 1-2 $\mathrm{fb}^{-1}$ data samples obtained from LHC $p p$ collisions at $\sqrt{s}=7.0 \mathrm{TeV}$.

The CDF and D0 experiments have annually produced combined Tevatron Higgs search results starting in 2006. The Higgs search is challenging due to the low expected signal yields and the large contributions from other SM backgrounds in the final states used for the Higgs searches. In each analysis iteration substantial effort is made to improve overall signal acceptance by, for example, loosening lepton and $b$ hadron identification requirements, adding backup triggers which select events online using different aspects of the signal signature, and relaxing kinematic selections. As signal acceptance is improved, the background contributions to our search topologies similarly increase and become more challenging to model. As much as possible, events are separated into categories with similar $S / \sqrt{B}$ to preserve the large contribution to the overall search sensitivity originating from the highest $S / \sqrt{B}$ events. Prior to consideration of detector acceptance and event selection efficiencies, the $10 \mathrm{fb}^{-1} \mathrm{CDF}$ and D0 datasets potentially contain about 500 Higgs boson events in the main search channels $\left(H \rightarrow W^{+} W^{-}, W H \rightarrow l v b \bar{b}, Z H \rightarrow v v b \bar{b}\right.$, and $\left.Z H \rightarrow l^{+} l^{-} b \bar{b}\right)$ for potential Higgs boson masses $\left(m_{H}\right)$ in the range between 115 and $185 \mathrm{GeV} / c^{2}$.

\section{Low mass SM Higgs searches}

Tevatron searches for a "low mass" $\left(m_{H}<135 \mathrm{GeV} / c^{2}\right)$ SM Higgs boson are discussed in some detail within these proceedings for both the primary $(H \rightarrow b \bar{b})[4]$ and secondary $\left(H \rightarrow \tau^{+} \tau^{-}\right.$ and $H \rightarrow \gamma \gamma$ ) [5] search channels. Here we summarize the features of our most sensitive low mass Higgs search channels. A low mass Higgs boson preferentially decays $H \rightarrow b \bar{b}$. It is most easily identified in events produced via associated Higgs production, $W H$ and $Z H$, when the $W$ and $Z$ decay leptonically, into final states of $W H \rightarrow l v b \bar{b}, Z H \rightarrow v v b \bar{b}$, and $Z H \rightarrow l^{+} l^{-} b \bar{b}$ where $\ell=e$ or $\mu$. In events with a reconstructed $W$ or $Z$ boson and two or more additional jets, the dijet invariant mass is used to search for a resonance originating from the $H \rightarrow b \bar{b}$ decay. To reduce the background from $W$ and $Z$ production in association with jets, $b$-jets are "tagged" by identifying the secondary vertex or tracks with high impact parameter originating from a $B$ hadron decay. The 
dijet mass spectrum is shown in Fig. 1 for the inclusive sample of $W H \rightarrow \ell v b \bar{b}$ candidate events as well as the subsets of these events with one and two $b$-tagged jets. Search sensitivity is greatly improved by categorizing events according to the number of $b$-tags and the purity of the applied $b$-tagging algorithms.
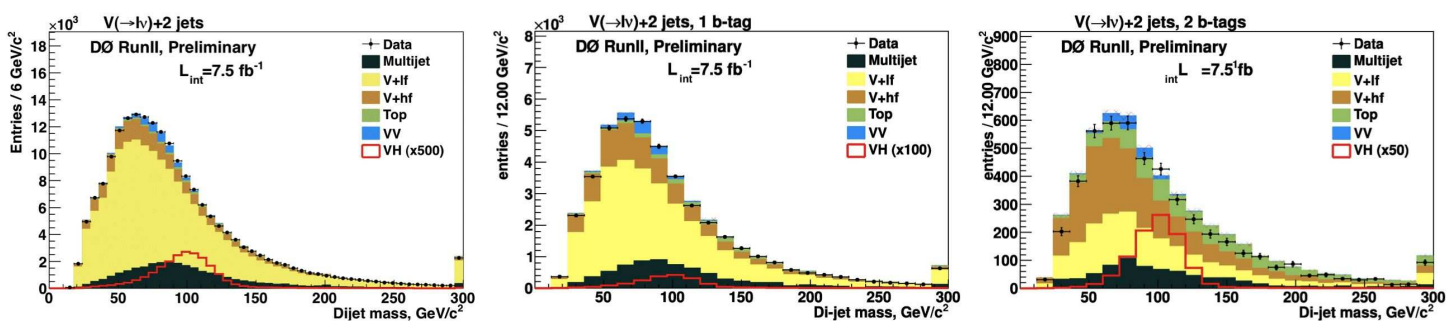

Figure 1: Dijet mass spectra for $W H \rightarrow \ell v b \bar{b}$ candidate events for the inclusive sample (left), subset with one $b$-tag (middle), and subset with two $b$-tags (right). As additional tagging requirements are made the $W+$ jets background is greatly reduced, while the $W H$ signal and heavy flavor backgrounds $(W+b \bar{b} / c \bar{c}$ and $t \bar{t}$ ) become more prominent.

\section{High mass SM Higgs searches}

Tevatron searches for a "high mass" Higgs boson $\left(m_{H}>135 \mathrm{GeV} / c^{2}\right)$ are also discussed in some detail separately within these proceedings $[6,7]$. The primary search channel at the Tevatron is $g g \rightarrow H \rightarrow W^{+} W^{-} \rightarrow \ell^{+} v \ell^{-} v$ due to the significant gluon fusion production cross section and easily identifiable final state. The high mass analyses benefit from separating events into categories according to the number of jets and the number of leptons to take advantage of changes in event kinematics that result from the different, dominant signal and background processes associated with each final state. Cross sections and uncertainties for the dominant signal production process, $g g \rightarrow H$ in particular, are obtained from state of the art NNLL and NNLO calculations as explained in Ref. [8]. Since there are neutrinos in the final state, the invariant mass of the Higgs boson cannot be reconstructed. The best variable for distinguishing $H \rightarrow W W$ from the background is the separation between the charged leptons, $\mathrm{dR}=\sqrt{d \eta^{2}+d \phi^{2}}$, which differs for the spin-zero $H \rightarrow W W$ and spin-one $Z \rightarrow W W$ decays as illustrated in Fig. 2. Also shown is the equivalent plot for same sign leptons, one of the control regions used to test background modeling.

\section{Validation of multivariate analysis techniques}

Multivariate techniques are used in most Tevatron SM Higgs boson searches to maximize their sensitivity to a potential signal. The main techniques used are Neural Networks, Matrix Element probabilities, and Boosted Decision Trees. By taking advantage of the different correlations among multiple kinematic input variables for signal and background processes, these approaches typically improve search sensitivities on the order of $10-20 \%$ with respect to optimized, cut-based analyses. Validation of these techniques is typically done by using them to measure higher statistics SM processes that contribute events in the same final states used for our Higgs searches. Two measurements of this type are illustrated in Fig. 3. The left plot shows a boosted decision tree discriminant 

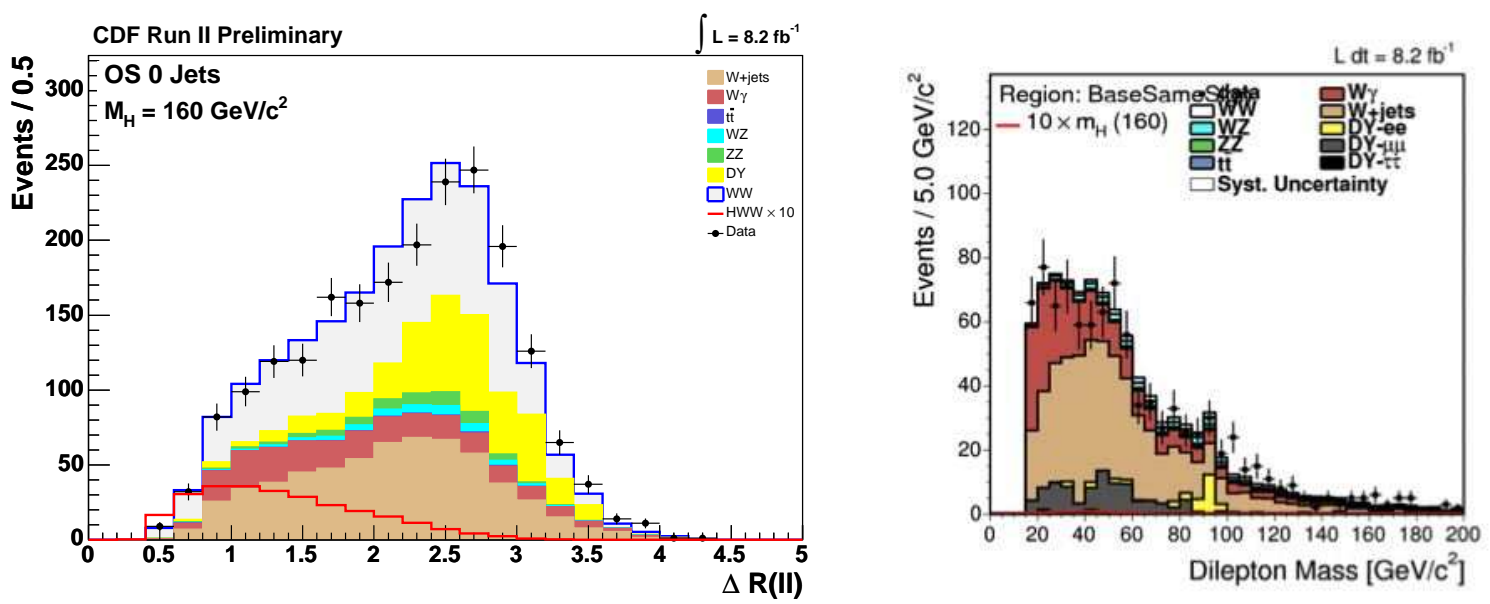

Figure 2: $\Delta \mathrm{R}$ distribution for opposite sign (OS) dilepton events with no accompanying jets (left) and for the same-sign-lepton control region used for testing modeling of the $W+$ jet fake background (right).

used by $\mathrm{D} 0$ to measure the cross section for SM $W Z+Z Z$ production in the same $v v b \bar{b}$ final used for $Z H$ Higgs searches. The right plot shows a neural network discriminant used in a CDF search for SM ZZ production in the same $\ell^{+} v \ell^{-} v$ final states used for $H \rightarrow W^{+} W^{-}$searches. In both cases, the measured SM production cross sections are found to be in good agreement with theoretical predictions demonstrating the robustness of the analysis techniques. A more complete review of Tevatron diboson measurements obtained from our Higgs search channels is contained within these proceedings [9].


Figure 3: Boosted decision tree discriminant used in a D0 diboson search for $W Z / Z Z \rightarrow v v b \bar{b}$ production (left) and a neural network discriminant used in a CDF diboson search for $Z Z \rightarrow \ell^{+} \ell^{-} v v$ production (left).

\section{Combined SM Higgs search}

Combined SM Higgs boson searches from CDF [10] and D0 [11] are described elsewhere within these proceedings. The two experiments additionally perform a joint SM Higgs search where correlations between shared systematic uncertainties are considered. The resulting combined 
limits on Higgs production are shown in Fig. 4. The 95\% C.L. upper limit on the cross section divided by the SM expectation is shown on the y-axis and is equal to one when SM sensitivity is achieved. The dotted line shows the expected upper cross section limit based on background-only pseudo-experiments, allowing systematic uncertainties to be fit within each pseudo-experiment and extracting the maximum signal contribution that can be accommodated at the $95 \%$ C.L. The green and yellow bands represent 1 and $2 \sigma$ variations in the limits from individual psuedo-experiments. The solid line is the upper limit observed in the Tevatron data.
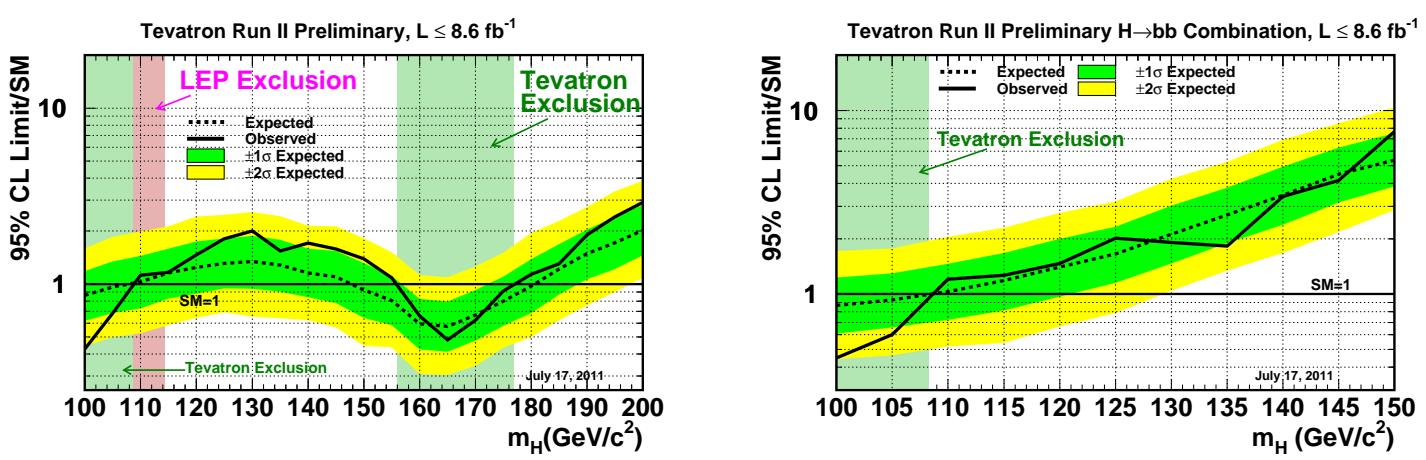

Figure 4: Combination of CDF and D0 searches for a SM Higgs boson for all channels (left) and $H \rightarrow b \bar{b}$ channels only (right).

The observed Higgs mass exclusion range is $157<m_{H}<177 \mathrm{GeV} / c^{2}$ which is somewhat smaller than the expected exclusion range of $148<m_{H}<181 \mathrm{GeV} / c^{2}$. The observed limits are found to be higher than the median, background-only expectation at the one sigma level for the mass range between 125 and $155 \mathrm{GeV} / c^{2}$. This excess is also consistent with the signal plus background expectation for a SM Higgs boson with $m_{H} \sim 130 \mathrm{GeV} / c^{2}$. The combined search also excludes Higgs boson masses below the current LEP Limits in the mass range between 100 and $109 \mathrm{GeV} / c^{2}$, consistent with expectations. At $115 \mathrm{GeV} / c^{2}$, just above the LEP limit, the expected (observed) exclusion is $1.16 * \mathrm{SM}(1.17 * \mathrm{SM})$. For $m_{H}=165 \mathrm{GeV} / c^{2}$, where the high mass exclusion is strongest, the $H \rightarrow W^{+} W^{-}$search channel dominates, but for $m_{H}=115 \mathrm{GeV} / c^{2}$, sensitivity comes from the combination of many independent analyses (see Table 1). The result of the combined Tevatron search based solely on our most sensitive $H \rightarrow b \bar{b}$ analysis channels is also shown in Fig. 4.

We can collectively view the dozens of discriminant outputs from the multiple search channels by gathering the histogram bins from all analysis channels, summing the contributions of those with similar $S / B$, and plotting them from lowest to highest $S / B$. The distribution is shown for $m_{H}=165 \mathrm{GeV} / c^{2}$ and $m_{H}=115 \mathrm{GeV} / c^{2}$ in Fig. 5. For the Higgs mass hypothesis of $165 \mathrm{GeV} / c^{2}$ the data clearly prefer the background-only model, while for the $m_{H}=115 \mathrm{GeV} / c^{2}$ hypothesis an excess of events is seen in the highest $S / B$ bins and a deficit of events is seen in slightly lower $S / B$ bins, resulting in the observed limit being roughly equal to the expected limit. 
Table 1: Tevatron analyses ordered by the expected upper limit at 95\% CL for a Higgs boson mass hypothesis of $115 \mathrm{GeV} / c^{2}$.

\begin{tabular}{cccc}
\hline \hline $\begin{array}{c}\text { Analysis } \\
\text { channel }\end{array}$ & Experiment & $\begin{array}{c}\text { Expected } \\
\text { limit } \sigma / \sigma_{S M} @ 115 \mathrm{GeV}\end{array}$ & $\begin{array}{c}\text { Integrated } \\
\text { Luminosity }\left(\mathrm{fb}^{-1}\right)\end{array}$ \\
\hline$W H \rightarrow \ell v b \bar{b}$ & $\mathrm{CDF}$ & 2.8 & 7.5 \\
$Z H / W H \rightarrow \mathbb{E}_{T} b \bar{b}$ & $\mathrm{CDF}$ & 3.0 & 7.8 \\
$W H \rightarrow \ell v b \bar{b}$ & $\mathrm{D} 0$ & 3.5 & 8.5 \\
$Z H \rightarrow \ell \ell b \bar{b}$ & $\mathrm{CDF}$ & 3.9 & 7.7 \\
$Z H / W H \rightarrow E_{T} b \bar{b}$ & $\mathrm{D} 0$ & 4.0 & 8.4 \\
$Z H \rightarrow \ell \ell b \bar{b}$ & $\mathrm{D} 0$ & 5.0 & 8.6 \\
$H \rightarrow W W \rightarrow \ell v \ell v$ & $\mathrm{CDF}$ & 7.9 & 8.2 \\
$H \rightarrow W W \rightarrow \ell v \ell v$ & $\mathrm{D} 0$ & 8.5 & 8.1 \\
$t t H$ & $\mathrm{CDF}$ & 11.7 & 7.5 \\
$H \rightarrow \gamma \gamma$ & $\mathrm{D} 0$ & 12.5 & 8.2 \\
$H \rightarrow \tau \tau$ & $\mathrm{CDF}$ & 12.6 & 8.3 \\
$H \rightarrow \tau \tau$ & $\mathrm{D} 0$ & 12.8 & 4.3 \\
$H \rightarrow \gamma \gamma$ & $\mathrm{CDF}$ & 13.5 & 7.0 \\
$Z H / W H \rightarrow q q b \bar{b}$ & $\mathrm{CDF}$ & 18.0 & 4.0 \\
$H \rightarrow W W \rightarrow \ell v q \bar{q}$ & $\mathrm{D} 0$ & 19.5 & 5.4 \\
$H \rightarrow Z Z$ & $\mathrm{CDF}$ & 82.8 & 8.2 \\
\hline Total & $\mathrm{CDF}+\mathrm{D} 0$ & 1.16 & 8.0 \\
\hline \hline
\end{tabular}
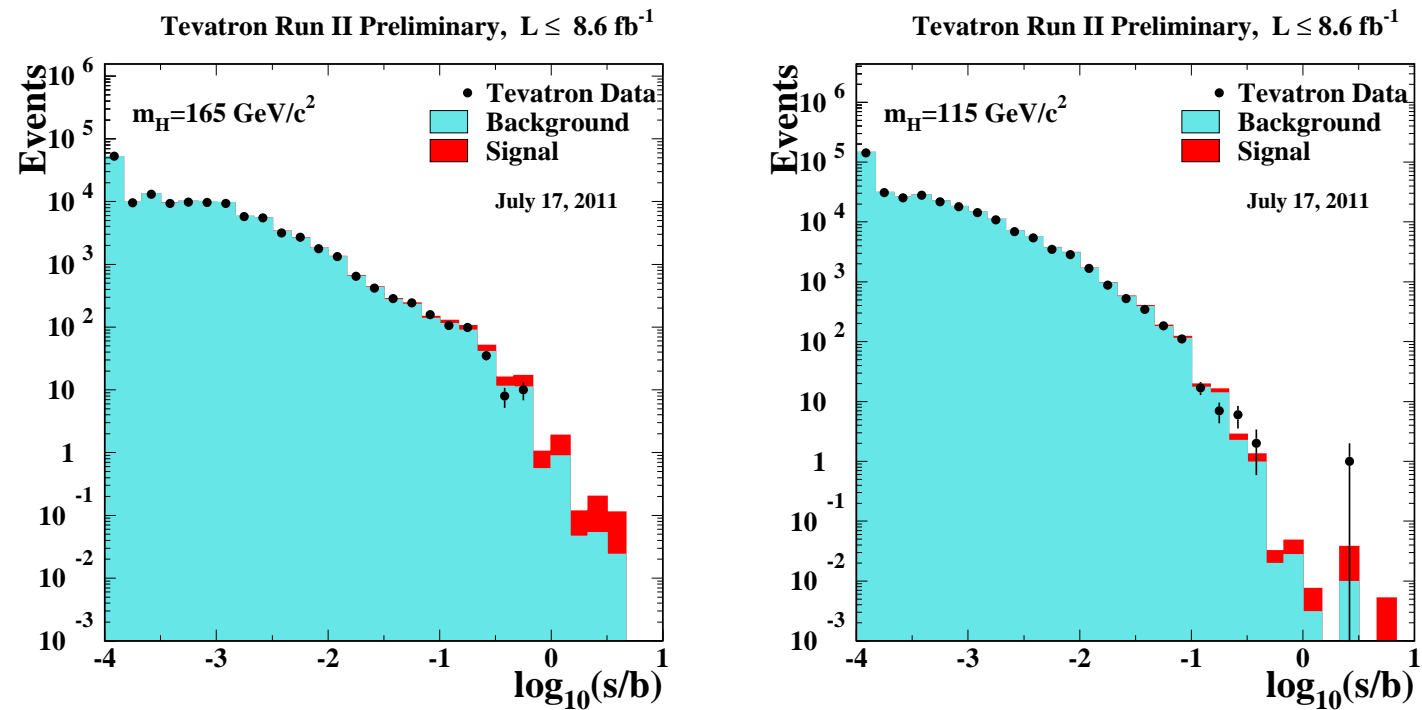

Figure 5: Data in all search channels sorted into bins of varying $S / B$ for Higgs mass hypotheses of $m_{H}=165 \mathrm{GeV} / c^{2}$ (left) and $m_{H}=115 \mathrm{GeV} / c^{2}$ (right). 


\section{Beyond SM Higgs searches}

SM Tevatron search limits are also interpreted in terms of limits on fourth generation and fermiophobic Higgs models. The presence of fourth generation quarks can enhance $g g \rightarrow H$ production by as much as a factor of nine and also modify Higgs branching ratios. In the case of a fermiophobic Higgs, production is limited to associated $W / Z$ boson and vector boson fusion mechanisms. Decays to fermions are forbidden and the dominant decay modes for low mass Higgs bosons are $H \rightarrow W^{+} W^{-}$and $H \rightarrow \gamma \gamma$. Tevatron limits based on these scenarios are shown in Fig. 6 .
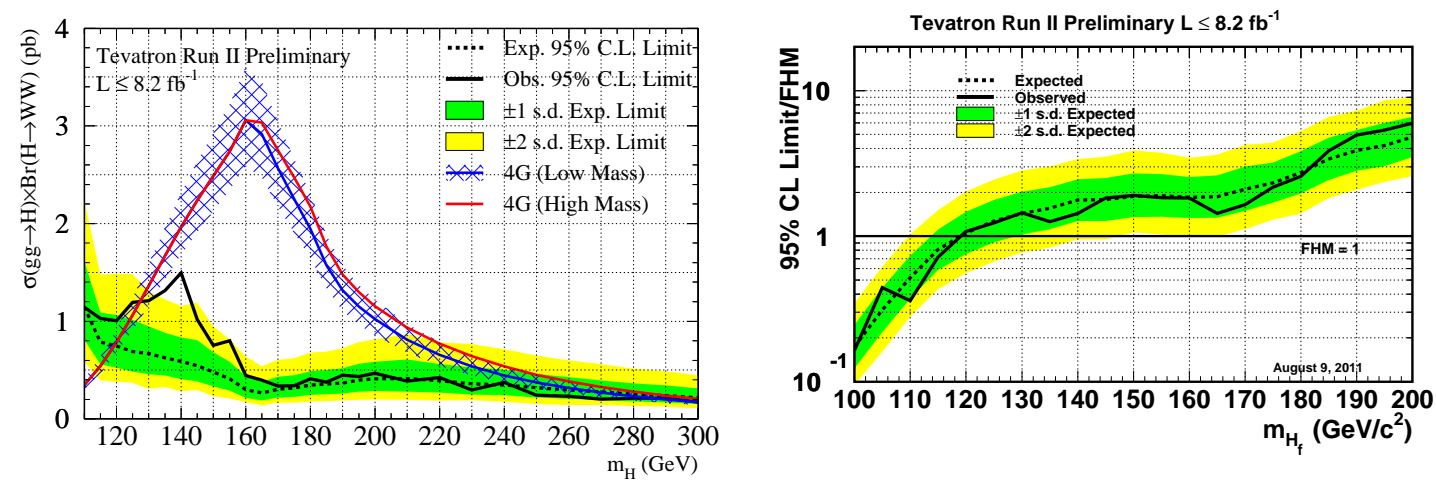

Figure 6: Combined Tevatron cross section limits from beyond SM Higgs searches based on fourth generation (left) and fermiophobic (right) models.

The Tevatron experiments also perform targeted searches for beyond SM Higgs production in several different scenarios including supersymmetry. These are presented in some detail elsewhere within these conference proceedings [12]. The MSSM predicts enhanced Higgs production cross sections and decays to down-type fermions for large values of $\tan \beta$, the ratio of the vacuum expectation value for up-type and down-type fermions, thereby enhancing contributions to the $H \rightarrow b \bar{b}$ and $H \rightarrow \tau \tau$ final states. Tevatron searches primarily use the invariant mass of the $b$-jets or the invariant mass of the visible $\tau$ decay products as discriminants and set limits close to the theoretically interesting value of $\tan \beta=30$, which is approximately the ratio of $m_{t}$ to $m_{b}$. Small excesses on the order of $2 \sigma$ (including trials factors) are observed in both the CDF and D0 $H+b \rightarrow b b+b$ search channels (Fig. 7).

\section{Outlook}

Figure 8 shows the expected a priori signal sensitivity as a function of mass and analyzed integrated luminosity per experiment. The projection incorporates a factor of 1.25 improvement in expected limits based on ongoing efforts to improve lepton identification efficiencies, b-tagging efficiencies, trigger selection, and jet energy resolution. Based on the full $10 \mathrm{fb}^{-1}$ Tevatron datasets which have now been collected, we project a $3 \sigma$ sensitivity to SM Higgs productions for a Higgs mass of $115 \mathrm{GeV} / c^{2}$. The sensitivity is projected to be at least $2.4 \sigma$ across the entire Higgs boson mass range between 100 to $185 \mathrm{GeV} / c^{2}$. 

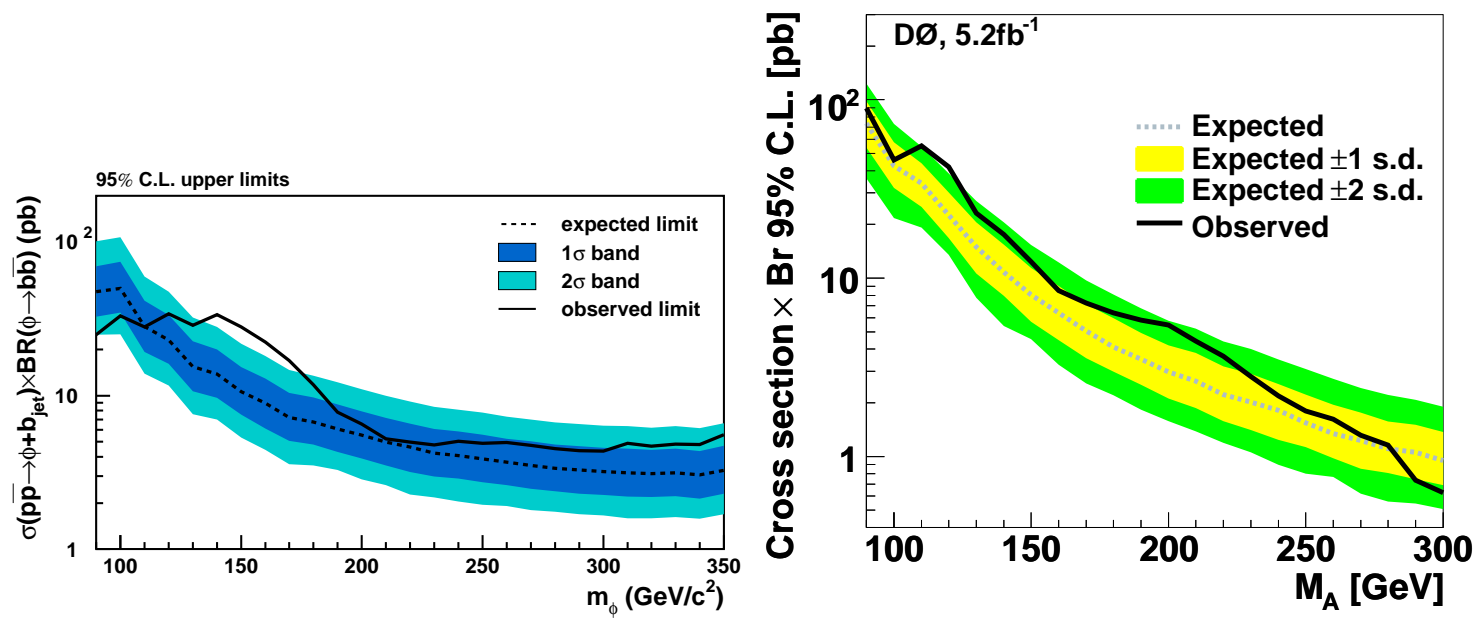

Figure 7: CDF (left) and D0 (right) cross section limits from $H+b \rightarrow b b+b$ MSSM Higgs search channel.

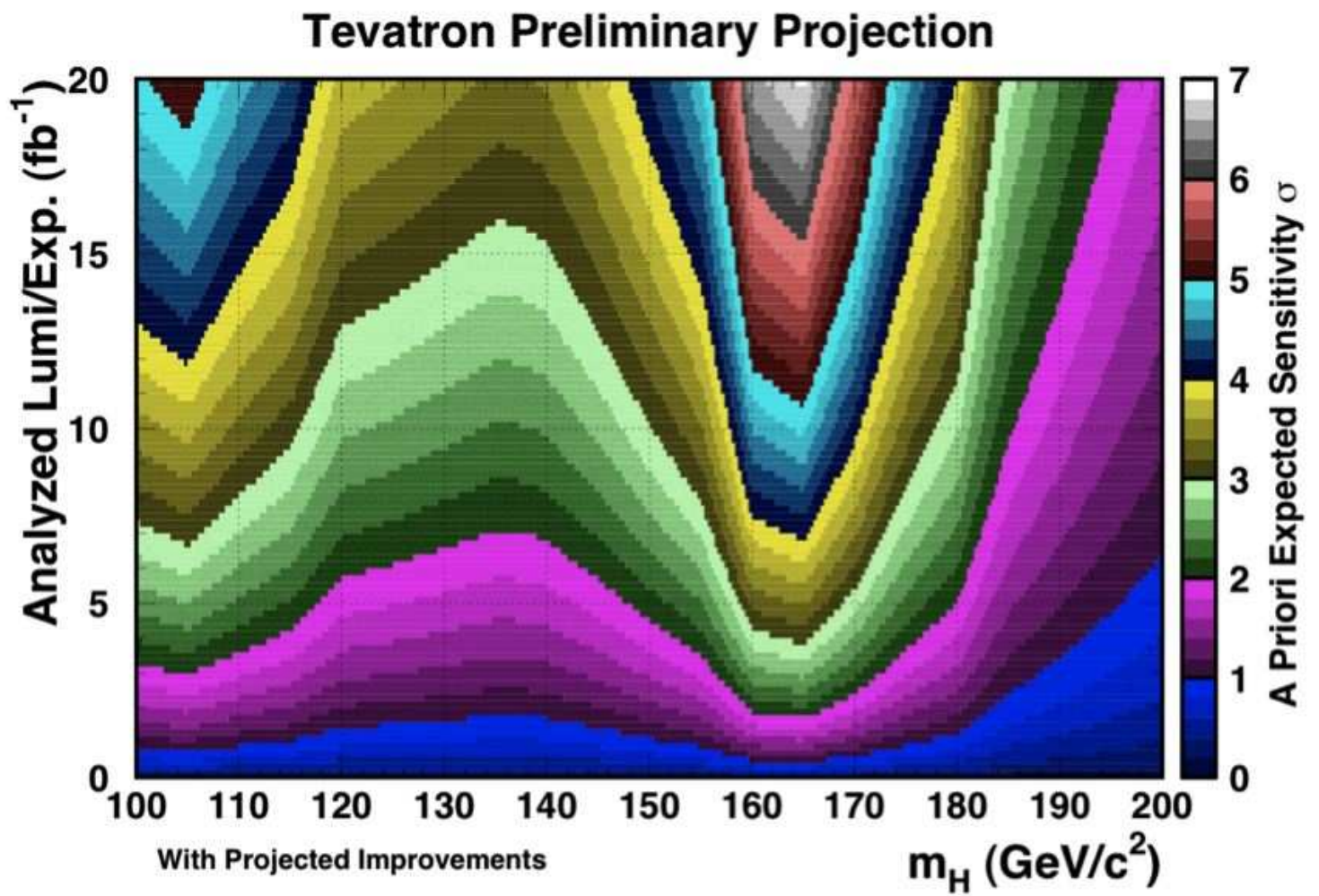

Figure 8: Projections of expected Higgs sensitivity, assuming a factor of 1.25 improvement in the expected limits at each mass point from more sophisticated analysis techniques. 


\section{Summary}

The CDF and D0 experiments at the Tevatron have a comprehensive and aggressive search program for the Higgs boson which can be divided into three main categories : high mass, low mass, and beyond SM searches. The newest high mass combination from the Tevatron excludes $157<$ $m_{H}<177 \mathrm{GeV} / c^{2}$ using datasets corresponding to an integrated luminosity of up to $8.2 \mathrm{fb}^{-1}$. In the low mass range, the combination of all channels gives a $1.16^{*} \mathrm{SM}\left(1.17^{*} \mathrm{SM}\right)$ expected (observed) exclusion for $m_{H}=115 \mathrm{GeV} / c^{2}$ and a $95 \%$ C.L. exclusion for the mass range between 100 and 109 $\mathrm{GeV} / c^{2}$. Tevatron MSSM Higgs searches set limits at theoretically motivated values of $\tan \beta$ and $H+b \rightarrow b b+b$ searches from both CDF and D0 contain interesting signal-like excesses at the 2 $\sigma$ level. With the $10 \mathrm{fb}^{-1}$ datasets collected by the Tevatron experiments, the final combination of Tevatron Higgs searches is projected to have at least $2.4 \sigma$ level sensitivity over the entire $100<$ $m_{H}<185 \mathrm{GeV} / c^{2}$ mass range.

\section{References}

[1] ALEPH, CDF, D0, DELPHI, L3, OPAL, and SLD Collaborations, LEP Electroweak Working Group, Tevatron Electroweak Working Group, and SLD ELectoweak and Heavy Flavor Groups, Precision Electroweak Measurements and Constraints on the Standard Model, CERN-PH-EP/2009-023, arXiv:0911.2604 [hep-ex];

[2] ALEPH, DELPHI, L3, and OPAL Collaborations and LEP Working Group for Higgs Boson Searches, Search for the Standard Model Higgs Boson at LEP, Phys. Lett. B 565, 61-75 (2003);

[3] W. Murray, Higgs searches at the LHC, PoS (EPS-HEP 2011);

[4] K. Potamianos, Search for the standard model Higgs boson in final states with b quarks at the Tevatron, PoS (EPS-HEP 2011);

[5] A. Kasmi, Search for the standard model Higgs boson in final states with photons or taus at the Tevatron, PoS (EPS-HEP 2011);

[6] B. Tuchming, Search for high mass standard model Higgs bosons at the Tevatron in the main channels, PoS (EPS-HEP 2011);

[7] A. Limosani, Other searches for a high mass Higgs boson at Tevatron, PoS (EPS-HEP 2011);

[8] T. Aaltonen et al.(CDF and D0 Collaborations), Combined CDF and DO Upper Limits on Standard Model Higgs boson production with up to $8.6 \mathrm{fb}^{-1}$ of Data, FERMILAB-CONF-11-354-E, arXiv:1107.5518 [hep-ex];

[9] T. Guillemin, Measurement of diboson production in lepton plus jet(s) decays at the Tevatron, $\mathrm{PoS}$ (EPS-HEP 2011);

[10] A. Buzatu, Combined upper limit on standard model Higgs boson production at CDF, PoS (EPS-HEP 2011);

[11] S. Grender, Combined upper limit on standard model Higgs boson production at D0, PoS (EPS-HEP 2011);

[12] F. Couderc, Searches for supersymmetric Higgs bosons at Tevatron, PoS (EPS-HEP 2011); 